\title{
Neuroendocrine Carcinoma of the Cervix with Sister Mary Joseph's Nodule: About a Case and Literature Review
}

\author{
Khadija Darif, Karima Oualla, Reda El Hazzaz, Othmane Zouiten, Oumaima Siyouri, Nisrine Acharfi, \\ Raihana Boujarnija, Lamiae Amaadour, Zineb Benbrahim, Samia Arifi, Nawfel Mellas
}

Medical Oncology Department, Hassan II University Hospital Center, Fez, Morocco

Email: darifk48@gmail.com

How to cite this paper: Darif, K., Oualla, K., El Hazzaz, R., Zouiten, O., Siyouri, O., Acharfi, N., Boujarnija, R., Amaadour, L., Benbrahim, Z., Arifi, S. and Mellas, N. (2020) Neuroendocrine Carcinoma of the Cervix with Sister Mary Joseph's Nodule: About a Case and Literature Review. Open Access Library Journal, 7: e6874.

https://doi.org/10.4236/oalib.1106874

Received: September 29, 2020

Accepted: November 17, 2020

Published: November 20, 2020

Copyright $\odot 2020$ by author(s) and Open Access Library Inc.

This work is licensed under the Creative Commons Attribution International License (CC BY 4.0).

http://creativecommons.org/licenses/by/4.0/

\begin{abstract}
Neuroendocrine tumors are a rare and aggressive histological variant of the cervix, and they have several differences compared to other types of cervical cancer, in prognosis, diagnosis and treatment. In this paper we report the case of a 39-year-old patient that was taken care of in the medical oncology department at the Hassan II University Hospital Center, for an advanced neuroendocrine carcinoma of the cervix with a Sister Mary Joseph's nodule. The aim of this work is to highlight this rare location of neuroendocrine tumors and their very aggressive character; by describing the different clinical, radiological and anatomopathological aspects of the primary tumor as well as umbilical metastasis.
\end{abstract}

\section{Subject Areas \\ Oncology}

\section{Keywords}

Neuroendocrine Carcinoma, Cervix, Sister Mary Joseph's Nodule, HPV

\section{Introduction}

Neuroendocrine tumors of the cervix represent a very rare histological type, which does not exceed $2 \%$ of all cervical tumors [1]. In literature the median age at diagnosis was 40 years old; there was also a higher proportion of Asian women with NECC (neuroendocrine carcinoma of the cervix), when compared to women with squamous cell carcinoma of the cervix [2]. The diagnosis is mainly based on histological and immunohistochemical data. They are distinguished 
from squamous cell carcinomas by their higher recurrence rate and their delayed diagnosis. Because NECC is uncommon, the etiology and predisposing risk factors are poorly understood, also the few cases revealed in the literature are not sufficient to define recommendations and therapeutic strategies. Treatment of NEC is usually more intensive than that for most other types of cervical cancer, and therapy often utilizes multiple different modalities such as surgery, chemotherapy and radiation. The prognosis depends on the stage but it is generally unfavorable when compared with the other subtypes.

Sister Marie-Josèphe's nodule is also an umbilical metastasis from an intra-abdominal tumor in most cases. It is a rare clinical sign with an incidence of $1 \%$ to $3 \%$ of all abdominopelvic neoplasms, and also has a poor prognosis.

In this article we do not only describe a rare primary tumor of the cervix, but also an exceptional secondary location: Sister Mary Joseph's nodule is a rare umbilical metastasis especially of the genitourinary and intestinal tract, and its discovery predicts a very advanced stage of the disease.

\section{Case Report}

We report the case of a 39 years old patient, G2P2, without any medical history, followed at the Hassan II University Hospital of Fez for an advanced cervical tumor, the patient had oliguria with renal colic for 4 months, she consulted in urology and a renal ultrasound was performed, which revealed a bilateral hydronephrosis upstream of a poorly limited pelvic tissue process, the assessment was completed by a pelvic CT, with discovery of a vegetative mass of the bladder floor invading the right ureter with significant uretero-hydnephrosis. A bladder tumor was initially suspected; the patient underwent a bilateral nephrostomy associated with an endoscopic resection of the bladder mass with a cervical biopsy; the anatomopathological examination was in favor of a small cell carcinoma; immunohistochemistry confirmed the diagnosis of a neuroendocrine carcinoma grade 3 with: high expression of synaptophysin and neuron-specific enolase (NSE) and weak expression of CD99, the chromogranin A and CD 56 were negative, Ki $67=95 \%$. HPV infection was not detected in this patient; she was referred in medical oncology to complete therapeutic care.

On the clinical examination: the patient had an ECOG performance status of 3 , the abdomen was very distended with collateral venous circulation, an umbilical nodule of $2 \mathrm{~cm}$, a pelvic tenderness to palpation, and a very painful vaginal touch with pelvic shielding (Figure 1).

Thoraco abdominopelvic CT scan objectified a bulky tissue mass extending from the cervix, invading the neighboring structures and measuring approximately $15 \times 14 \times 13 \mathrm{~cm}$ in diameter, this process invades the isthmus and the uterine body, the vagina, the bilateral parameters and comes into contact with colon and rectum without separation border; it also invades the bladder which is the seat of a tumor mass, in contact with the anterior abdominal wall. There was a presence of multiple iliac, bilateral lumbar-aortic, and bilateral coeliomenteric 
metastatic lymph nodes, Medium abundance ascites with peritoneal carcinosis masses of pelvic and metastatic umbilical nodes (Figure 2(a); Figure 2(b)).

The diagnosis of an advanced neuroendocrine carcinoma of the cervix was retained on radiological and anatomopathological criteria.

Unfortunately the patient died a few days after diagnosis, before starting any treatment.

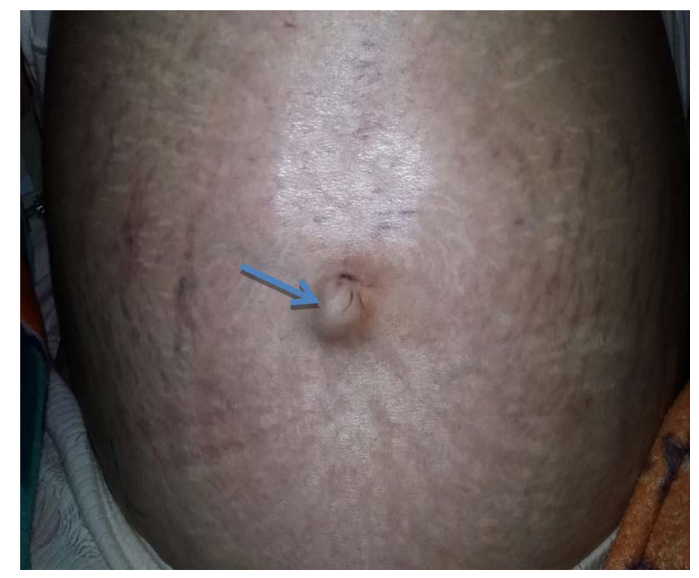

Figure 1. Metastatic cutaneous nodule at umbilicus.
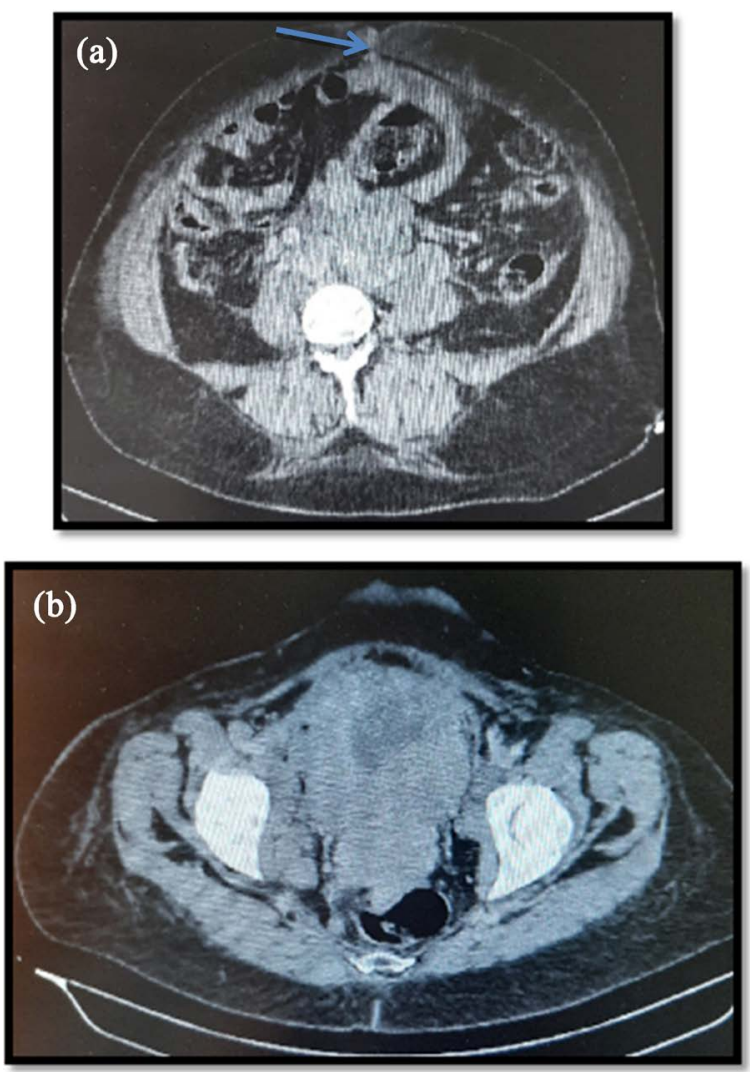

Figure 2. (a) Abdominal CT scan demonstrating metastatic umbilical nodule (Mary Joseph Sister nodule); (b) Pelvic CT scan revealing a bulky tissue mass extending from the cervix, invading in contact with rectum without separation border; and invades the bladder. 


\section{Discussion}

Besides being rare, neuroendocrine tumors are very aggressive malignant tumors with a poor prognosis compared to the usual histological types of the uterine cervix [2]. According to a systematic review of the literature published in 2018 by Clemens B. Tempfer, including 112 studies: The pooled proportion of Neuroendocrine carcinoma of the cervix among women with cervical cancer was $1.41 \%[3]$.

Neuroendocrine tumors originate from the embryonic neuroectoderm and exhibit an immunohistochemical profile which is consistent with endocrine glandular cells [3]. They may not secrete peptide hormones. They are usually located in the gastrointestinal tract, the pancreas and the lungs.

The histological grading of neuroendocrine tumors of the cervix is the same in the other locations which are classified into well-differentiated NEC and poorly differentiated NEC [4]. Well differentiated NECs include neuroendocrine tumors G1 (typical carcinoid) and NEC G2 (atypical carcinoid), and G3. Poorly differentiated neuroendocrine carcinomas include small cell NEC and large cell NEC [3] [5].

Although HPV is known to be a risk factor in the development of cervical carcinoma; in a recent meta-analysis which was published by Castel et al. [6], the HPV infection data were analyzed in 403 NECC cases of small cells; $85 \%$ of the cases were positive for HPV, HPV18 and HPV16 were the predominant HPV subtypes; hence the indication for prophylactic vaccination, however in our case, patient had a negative HPV profile.

Like all neuroendocrine tumors of the cervix, clinical symptoms depend on the extent of the disease; a paraneoplastic syndrome is very rare.

The immunohistochemical markers which are necessary for the diagnosis of all neuroendocrine tumors are: synaptophysin, chromogranin, CD56, and NSE; positive staining of at least two neuroendocrine markers is recommended to establish a diagnosis. In our case, the diagnosis was based on positivity of synaptophysin and NSE. Since cervical NECs are unusual, there are no randomized clinical trials to determine the optimal therapy for localized stages; The most common treatment modalities in the different series was radical surgery associated with neoadjuvant or adjuvant chemotherapy. Other regimens based on radiotherapy, or chemotherapy with cisplatin or radiotherapy followed by chemotherapy were also commonly used in several series. There was no retrospective or prospective comparison between the different treatment regimens. For relapses, chemotherapy has been used in most studies, followed by radiotherapy and surgery in a few series [3]. For patients with metastatic disease, systemic chemotherapy is recommended [7] [8]. Based on the chemotherapy regimens used in other localization of NE carcinoma, the cisplatin etoposide association is the adopted schema in advanced and metastatic forms [9] the other chemotherapy regimens that have been reported in the literature are cisplatin/irinotecan [10], carboplatin/paclitaxel [11], and cisplatin/vincristine/bleomycin [12]. 
The NECC are very aggressive and similar to small cell lung cancer, at the time of initial diagnosis, they are often locally advanced or metastasized. As in our case where patient presented at an advanced stage, with an umbilical metastasis; known as the "The sister mary joseph's nodule"; this lesion was first described in 1864 by the name of Sister Mary Joseph Dempsey (1856-1939), surgical assistant to Dr. William James Mayo, who first noticed the association between the malignant pelvic abdominals and metastatic umbilical nodules [13] [14]. It is a rare secondary lesion which does not exceed $3 \%$ of the gastrointestinal and genitourinary primitives, Some other rare locations are also described as the lung, lymphoma, breast, kidney, and in $30 \%$ of the cases the primitive can be unknown [15], the presence of this nodule is predictive of a poor prognosis.

The sister mary joseph's nodule presents clinically as a form of a non-sensitive palpable umbilical nodule of a color varying from purplish to reddish brown. We can sometimes observe ulcers or skin cracks with even a serious and purulent exudate. The diameter of the lesion rarely exceeds 5 centimeters [16].

Imaging can help clinical examination to characterize better the umbilical nodule; it sometimes allows the discovery of umbilical metastasis. The prognosis for patients with Sister Mary Joseph's nodule remains poor because it is a sign of advanced malignancy. Management must take into account the primitive tumor and which is often palliative.

Our patient was unfortunately diagnosed at a very advanced stage, thus she was unable to benefit from systemic chemotherapy. In the literature, no case of such a rare association has been described; the association of an advanced neuroendocrine tumor of the cervix with umbilical metastasis in a young patient in our case highlighted well the very aggressive character and the poor prognosis of this pathology.

\section{Conclusion}

Neuroendocrine tumors of the cervix are certainly rare tumors, but given their aggressiveness and poor prognosis, an adequate therapeutic strategy must be proposed especially in the early stages and perhaps patients should be included in therapeutic trials; the presence of the nodule of The Sister Mary Joseph's nodule does not change anything in the care of the patient but remains a good predictive of the evolution of the disease.

\section{Conflicts of Interest}

The authors declare no conflicts of interest regarding the publication of this paper.

\section{References}

[1] Albores-Saavedra, J., Larraza, O., Poucell, S. and Rodríguez Martínez, H.A. (1976) Carcinoid of the Uterine Cervix: Additional Observations on a New Tumor Entity. Cancer, 38, 2328-2342. https://doi.org/10.1002/1097-0142(197612)38:6<2328::AID-CNCR2820380620>3.0. $\underline{\mathrm{CO} ; 2-\mathrm{J}}$ 
[2] McCusker, M.E., Coté, T.R., Clegg, L.X. and Tavassoli, F.J. (2003) Endocrine Tumors of the Uterine Cervix: Incidence, Demographics, and Survival with Comparison to Squamous Cell Carcinoma. Gynecologic Oncology, 88, 333-339. https://doi.org/10.1016/S0090-8258(02)00150-6

[3] Tempfer, C.B., Tischoff, I., Dogan, A., Hilal, Z., Schultheis, B., Kern, P. and Rezniczek, G.A. (2018) Neuroendocrine Carcinoma of the Cervix: A Systematic Review of the Literature. BMC Cancer, 18, 530.

[4] Kim, J.Y., Hong, S.M. and Ro, J.Y. (2017) Recent Updates on Grading and Classification of Neuroendocrine Tumors. Annals of Diagnostic Pathology, 29, 11-16. https://doi.org/10.1016/j.anndiagpath.2017.04.005

[5] Gadducci, A., Carinelli, S. and Aletti, G. (2017) Neuroendrocrine Tumors of the Uterine Cervix: A Therapeutic Challenge for Gynecologic Oncologists. Gynecologic Oncology, 144, 637-646. https://doi.org/10.1016/j.ygyno.2016.12.003

[6] Castle, P.E., et al. (2018) A Systematic Review and Meta-Analysis on the Attribution of Human Papillomavirus (HPV) in Neuroendocrine Cancer of the Cervix. Gynecologic Oncology, 148, 422-429. https://doi.org/10.1016/j.ygyno.2017.12.001

[7] Gardner, G.J., Reidy-Lagunes, D. and Gehrig, P.A. (2011) Neuroendocrine Tumors of the Gynecologic Tract: A Society of Gynecologic Oncology (SGO) Clinical Document. Gynecologic Oncology, 122, 190-198.

https://doi.org/10.1016/j.ygyno.2011.04.011

[8] Satoh, T., Takei, Y., Treilleux, I., Devouassoux-Shisheboran, M., Ledermann, J., Viswanathan, A.N., Mahner, S., Provencher, D.M., Mileshkin, L., Åvall-Lundqvist, E., Pautier, P., Reed, N.S. and Fujiwara, K. (2014) Gynecologic Cancer InterGroup (GCIG) Consensus Review for Small Cell Carcinoma of the Cervix. International Journal of Gynecological Cancer, 24, S102-S108. https://doi.org/10.1097/IGC.0000000000000262

[9] Yin, Z.M., Yu, A.J., Wu, M.J., Fang, J., Liu, L.F., Zhu, J.Q. and Yu, H. (2015) Effects and Toxicity of Neoadjuvant Chemotherapy Preoperative Followed by Adjuvant Chemoradiation in Small Cell Neurdendocrine Cervical Carcinoma. European Journal of Gynaecological Oncology, 36, 326-329.

[10] Nasu, K., Hirakawa, T., Okamoto, M., Nishida, M., Kiyoshima, C., Matsumoto, H., Takai, N. and Narahara, H. (2011) Advanced Small Cell Carcinoma of the Uterine Cervix Treated by Neoadjuvant Chemotherapy with Irinotecan and Cisplatin Followed by Radical Surgery. Rare Tumors, 3, e6. https://doi.org/10.4081/rt.2011.e6

[11] Hoskins, P.J., Swenerton, K.D., Pike, J.A., Lim, P., Aquino-Parsons, C., Wong, F. and Lee, N. (2003) Small-Cell Carcinoma of the Cervix: Fourteen Years of Experience at a Single Institution Using a Combined-Modality Regimen of Involved-Field Irradiation and Platinum-Based Combination Chemotherapy. Journal of Clinical Oncology, 21, 3495-3501. https://doi.org/10.1200/JCO.2003.01.501

[12] Bermúdez, A., Vighi, S., García, A. and Sardi, J. (2001) Neuroendocrine Cervical Carcinoma: A Diagnostic and Therapeutic Challenge. Gynecologic Oncology, 82, 32-39. https://doi.org/10.1006/gyno.2001.6201

[13] Tso, S., Brockley, J., Recica, H. and Ilchyshy, A. (2013) Sister Mary Joseph's Nodule: An Unusual But Important Physical Finding Characteristic of Widespread Internal Malignancy. British Journal of General Practice, 63, 551-552. https://doi.org/10.3399/bjgp13X673900

[14] Quenu, L. (1896) Secondary Cancer in the Umbilicus. Revue de Chirurgie, 16, 97-133.

[15] Gabriele, R., Conte, M., Egidi, F. and Borghese, M. (2005) Umbilical Metastasis: 
Current Viewpoint. World Journal of Surgical Oncology, 3, Article No. 13.

[16] Schoijet, I.M., Rojas, A.A., Cortés, C. and Varela, C.U. (2018) Sister Mary Joseph's Nodule: From the History to the Images. A Case-Based Literature Review. Acta Gastroenterologica Latinoamericana, 48, 82-89. 